\title{
Circular-hyperbolic Fibonacci quaternions
}

\author{
Fügen Torunbalcı Aydın \\ Yildiz Technical University \\ Faculty of Chemical and Metallurgical Engineering \\ Department of Mathematical Engineering \\ Davutpasa Campus, 34220, Esenler, Istanbul, Turkey \\ e-mails: faydin@yildiz.edu.tr, ftorunay@gmail.com
}

Received: 19 July 2019

Revised: 21 April 2020

Accepted: 25 April 2020

\begin{abstract}
In this paper, circular-hyperbolic Fibonacci numbers and quaternions are defined. Also, some algebraic properties of circular-hyperbolic Fibonacci numbers and quaternions which are connected with circular-hyperbolic numbers and Fibonacci numbers are investigated. Furthermore, Honsberger's identity, the generating function, Binet's formula, d'Ocagne's identity, Cassini's identity, and Catalan's identity for these quaternions are given.
\end{abstract}

Keywords: Fibonacci number, Hyperbolic number, Dual-hyperbolic number, Circular-hyperbolic number, Circular-hyperbolic Fibonacci number.

2010 Mathematics Subject Classification: 11B37, 20G20, 11R52.

\section{Introduction}

The real quaternions were first described by Irish mathematician William Rowan Hamilton in 1843. Hamilton [9] introduced a set of real quaternions which can be represented as

$$
H=\left\{q=q_{0}+i q_{1}+j q_{2}+k q_{3} \mid q_{0}, q_{1}, q_{2}, q_{3} \in \mathbb{R}\right\}
$$

where

$$
i^{2}=j^{2}=k^{2}=-1, \quad i j=-j i=k, \quad j k=-k j=i, \quad k i=-i k=j .
$$

The real quaternions constitute an extension of complex numbers into a four-dimensional space and can be considered as four-dimensional vectors, in the same way that complex numbers are considered as two-dimensional vectors. Horadam $[10,11]$ defined complex Fibonacci and Lucas quaternions as follows: 


$$
\begin{aligned}
& Q_{n}=F_{n}+F_{n+1} i+F_{n+2} j+F_{n+3} k, \\
& K_{n}=L_{n}+L_{n+1} i+L_{n+2} j+L_{n+3} k,
\end{aligned}
$$

where $F_{n}$ and $L_{n}$ denote the $n$-th Fibonacci and Lucas numbers, respectively. Also, the imaginary quaternion units $i, j, k$ have the following rules

$$
i^{2}=j^{2}=k^{2}=-1, \quad i j=-j i=k, \quad j k=-k j=i, \quad k i=-i k=j .
$$

The studies that follows is based on the work of Horadam $[8,19,20]$.

The dual numbers are elements of the 2-dimensional real algebra

$$
\mathbb{D}=\left\{z=x+\varepsilon y \mid \varepsilon \neq 0, \varepsilon^{2}=0, x, y \in \mathbb{R}\right\}
$$

generating by 1 and $\varepsilon,[4,21]$. The work on the dual Fibonacci numbers and quaternions can be found in $[19,20]$.

Hyperbolic numbers have applications in different areas of mathematics and theoretical physics. The work on the hyperbolic numbers can be found in $[1,2,6,18,22,23,26]$. The set of hyperbolic numbers $\mathbb{H}$ can be described in the form as

$$
\mathbb{H}=\left\{z=x+h y \mid h \notin \mathbb{R}, h^{2}=1, x, y \in \mathbb{R}\right\}
$$

Majernik has introduced the multicomponent number system $[15,16]$. There are three types of the four-component number systems which have been constructed by joining the complex, binary and dual two-component numbers. Later, Farid Messelmi has defined the algebraic properties of the dual-complex numbers in the light of this study [17].

Dual-hyperbolic numbers $[1,3] w$ can be expressed in the form as

$$
\mathbb{D H}=\left\{w=z_{1}+\varepsilon z_{2} \mid z_{1}, z_{2} \in \mathbb{H}, \text { where } j^{2}=1, \varepsilon^{2}=0, \varepsilon \neq 0(j \varepsilon)^{2}=0\right\} .
$$

Here if $z_{1}=x_{1}+x_{2} j$ and $z_{2}=y_{1}+y_{2} j$, then any dual-hyperbolic number can be written

$$
\begin{gathered}
w=\left(x_{1}+x_{2} j\right)+\varepsilon\left(y_{1}+y_{2} j\right) \\
j^{2}=1, \quad \varepsilon \neq 0, \quad \varepsilon^{2}=0, \quad \varepsilon j=j \varepsilon, \quad(j \varepsilon)^{2}=0 .
\end{gathered}
$$

where $\in \mathbb{H}$ is set of hyperbolic numbers.

In 2019, Cihan, Azak, Güngör and Tosun [7] have defined dual-hyperbolic Fibonacci and Lucas numbers respectively, as follows

$$
\begin{aligned}
& D H F_{n}=F_{n}+F_{n+1} j+F_{n+2} \varepsilon+F_{n+3} j \varepsilon \\
& D H L_{n}=L_{n}+L_{n+1} j+L_{n+2} \varepsilon+L_{n+3} j \varepsilon
\end{aligned}
$$

where $F_{n}$ and $L_{n}$ denote the $n$-th Fibonacci and Lucas numbers, respectively. Also, the imaginary quaternion units $j, \varepsilon, j \varepsilon$ have the following rules

$$
j^{2}=1, \quad \varepsilon j=j \varepsilon, \quad \varepsilon^{2}=(j \varepsilon)^{2}=0 .
$$


Furthermore, some algebraic properties are proven for these numbers in [3]. Circular-hyperbolic numbers [5] $w$ can be expressed in the form as

$$
\mathbb{C H}=\left\{w=z_{1}+z_{2} h \mid z_{1}, z_{2} \in \mathbb{C}, \quad i^{2}=-1, h^{2}=1, h \neq \pm 1, \quad(i h)^{2}=1\right\},
$$

where $\in \mathbb{C}$ is set of complex numbers. Here if $z_{1}=x_{1}+i x_{2}$ and $z_{2}=y_{1}+i y_{2}$, then any circular-hyperbolic number can be written

$$
\begin{gathered}
w=\left(x_{1}+i x_{2}\right)+\left(y_{1}+i y_{2}\right) h \\
h^{2}=1, \quad h \neq \pm 1, \quad i h=-h i, \quad(i h)^{2}=1 .
\end{gathered}
$$

Addition, subtraction and multiplication of any two circular-hyperbolic numbers $w_{1}$ and $w_{2}$ are defined by

$$
\begin{aligned}
w_{1} \pm w_{2} & =\left(z_{1}+z_{2} h\right) \pm\left(z_{3}+z_{4} h\right)=\left(z_{1} \pm z_{3}\right)+\left(z_{2} \pm z_{4}\right) h \\
w_{1} \times w_{2} & =\left(z_{1}+z_{2} h\right) \times\left(z_{3}+z_{4} h\right)=\left(z_{1} z_{3}+z_{2} z_{4}\right)+\left(z_{1} z_{4}+z_{2} z_{3}\right) h .
\end{aligned}
$$

On the other hand, the division of two circular-hyperbolic numbers are given by

$$
\begin{aligned}
\frac{w_{1}}{w_{2}} & =\frac{z_{1}+z_{2} h}{z_{3}+z_{4} h} \\
\frac{\left(z_{1}+z_{2} h\right)\left(z_{3}-z_{4} h\right)}{\left(z_{3}+z_{4} h\right)\left(z_{3}-z_{4} h\right)} & =\frac{\left(z_{1} z_{3}-z_{2} z_{4}\right)}{z_{3}^{2}-z_{4}^{2}}+\frac{\left(z_{2} z_{3}-z_{1} z_{4}\right)}{z_{3}^{2}-z_{4}^{2}} h .
\end{aligned}
$$

If $\operatorname{Re}\left(w_{2}\right) \neq 0$,then the division $\frac{w_{1}}{w_{2}}$ is possible. The circular-hyperbolic numbers are defined by the basis $\{1, i, h, i h\}$. The base elements of the circular-hyperbolic numbers satisfy the following commutative multiplication scheme (Table 1).

\begin{tabular}{|c||r|r|r|r|}
\hline \hline$x$ & 1 & $i$ & $h$ & $i h$ \\
\hline \hline 1 & 1 & $i$ & $h$ & $i h$ \\
$i$ & $i$ & -1 & $i h$ & $-h$ \\
$h$ & $h$ & $-i h$ & 1 & $-i$ \\
$i h$ & $i h$ & $h$ & $i$ & 1 \\
\hline \hline
\end{tabular}

Table 1. Multiplication scheme of circular-hyperbolic numbers

The circular-hyperbolic numbers, just like quaternions, are a generalization of complex hyperbolic numbers by means of entities specified by four-component numbers. But hyperbolic and dual-hyperbolic numbers are commutative, whereas, circular-hyperbolic numbers are non-commutative. Moreover, the multiplication of these numbers gives the circular-hyperbolic numbers. Five different conjugations can operate on circular-hyperbolic numbers [5] as follows: 


$$
\begin{aligned}
& w=x_{1}+i x_{2}+h y_{1}+i h y_{2}, \\
& w^{*_{1}}=\left(x_{1}-i x_{2}\right)+\left(y_{1}-i y_{2}\right) h=z_{1}^{*}+z_{2}^{*} h, \text { complex conjugation, } \\
& w^{*_{2}}=\left(x_{1}+i x_{2}\right)-\left(y_{1}+i y_{2}\right) h=z_{1}-z_{2} h \text {, hyperbolic conjugation, } \\
& w^{* 3}=\left(x_{1}-i x_{2}\right)-\left(y_{1}-i y_{2}\right) h=z_{1}^{*}-z_{2}^{*} h \text {, coupled conjugation, } \\
& w^{* 4}=\left(x_{1}-i x_{2}\right)-\left(1-\frac{y_{1}+i y_{2}}{x_{1}+i x_{2}} h\right)=z_{1}^{*}-\left(1-\frac{z_{2}}{z_{1}} h\right), \\
& \text { complex-hyperbolic conjugation, } \\
& w^{* 5}=\left(y_{1}+i y_{2}\right)-\left(x_{1}-i x_{2}\right) h=z_{2}-z_{1}^{*} h \text {, anti-hyperbolic conjugation. }
\end{aligned}
$$

Therefore, the norm of the circular-hyperbolic numbers is defined as

$$
\begin{aligned}
& N_{w}^{*_{1}}=\left\|w \times w^{*_{1}}\right\|=\sqrt{\left|z_{1}\right|^{2}+\left|z_{2}\right|^{2}+2 \varepsilon \operatorname{Re}\left(z_{1} z_{2}{ }^{*}\right) h}, \\
& N_{w}^{*_{2}}=\left\|w \times w^{*_{2}}\right\|=\sqrt{z_{1}^{2}-z_{2}^{2}}, \\
& N_{w}^{*_{3}}=\left\|w \times w^{*_{3}}\right\|=\sqrt{\left|z_{1}\right|^{2}-\left|z_{2}\right|-2 i \operatorname{Im}\left(z_{1} z_{2}{ }^{*}\right) h}, \\
& N_{w}^{*_{4}}=\left\|w \times w^{*_{4}}\right\|=\sqrt{\left|z_{1}\right|^{2}-\left(z_{1}-\frac{z_{2}^{2}}{z_{1}}\right)+z_{1}^{*} z_{2} h}, \\
& N_{w}^{*_{5}}=\left\|w \times w^{*_{5}}\right\|=\sqrt{z_{2}\left(2 i \operatorname{Im} z_{1}\right)-\left(\left|z_{1}\right|^{2}-z_{2}^{2}\right) h} .
\end{aligned}
$$

In this paper, the circular-hyperbolic Fibonacci numbers and Fibonacci quaternions will be defined. In addition, the Honsberger identity, the d'Ocagne's identity, the generating function, Binet's formula, Cassini's identity, Catalan's identity for these quaternions are given.

\section{The circular-hyperbolic Fibonacci quaternions}

The circular-hyperbolic Fibonacci and Lucas quaternions can be defined by the basis $\{1, i, h, i h\}$, where $i, h$ and $i h$ satisfy the conditions

$$
i^{2}=-1 \quad h \neq \pm 1, \quad h^{2}=1, \quad i h=-h i \quad(i h)^{2}=1
$$

as follows

$$
\begin{aligned}
\mathbb{C H} F_{n} & =\left(F_{n}+i F_{n+1}\right)+\left(F_{n+2}+i F_{n+3}\right) h \\
& =F_{n}+i F_{n+1}+h F_{n+2}+i h F_{n+3}
\end{aligned}
$$

and

$$
\begin{aligned}
\mathbb{C H} L_{n} & =\left(L_{n}+i L_{n+1}\right)+\left(L_{n+2}+i L_{n+3}\right) h \\
& =L_{n}+i L_{n+1}+h L_{n+2}+i h L_{n+3} .
\end{aligned}
$$

The addition, subtraction and multiplication by real scalars of two circular-hyperbolic Fibonacci quaternions gives the circular-hyperbolic Fibonacci quaternion. Then, the addition and subtraction of the circular-hyperbolic Fibonacci quaternions are defined by

$$
\begin{aligned}
\mathbb{C H} F_{n} \pm \mathbb{C H} F_{m}= & \left(F_{n} \pm F_{m}\right)+i\left(F_{n+1} \pm F_{m+1}\right) \\
& +h\left(F_{n+2} \pm F_{m+2}\right)+i h\left(F_{n+3} \pm F_{m+3}\right) .
\end{aligned}
$$


The multiplication of a circular-hyperbolic Fibonacci quaternion by the real scalar $\lambda$ is defined as

$$
\lambda \mathbb{C H} F_{n}=\lambda F_{n}+i \lambda F_{n+1}+h \lambda F_{n+2}+i h \lambda F_{n+3} .
$$

By using Table 1, the multiplication of two circular-hyperbolic Fibonacci quaternions is defined by

$$
\begin{aligned}
\mathbb{C H} F_{n} \times \mathbb{C H} F_{m}= & \left(F_{n} F_{m}-F_{n+1} F_{m+1}+F_{n+2} F_{m+2}+F_{n+3} F_{m+3}\right) \\
& +i\left(F_{n+1} F_{m}+F_{n} F_{m+1}-F_{n+2} F_{m+3}+F_{n+3} F_{m+2}\right) \\
& +h\left(F_{n} F_{m+2}-F_{n+1} F_{m+3}+F_{n+2} F_{m}+F_{n+3} F_{m+1}\right) \\
& +i h\left(F_{n+1} F_{m+2}+F_{n} F_{m+3}+F_{n+3} F_{m}-F_{n+2} F_{m+1}\right) \\
\neq & \mathbb{C H} F_{m} \times \mathbb{C H} F_{n} .
\end{aligned}
$$

Also, there exits five conjugations as follows:

$$
\begin{aligned}
& \mathbb{C H} F_{n}^{*_{1}}=F_{n}-i F_{n+1}+h F_{n+2}-i h F_{n+3}, \quad \text { complex conjugation, } \\
& \mathbb{C H} F_{n}^{*_{2}}=F_{n}+i F_{n+1}-h F_{n+2}-i h F_{n+3}, \quad \text { hyperbolic conjugation, } \\
& \mathbb{C H} F_{n}^{*_{3}}=F_{n}-i F_{n+1}-h F_{n+2}+i h F_{n+3}, \quad \text { coupled conjugation, } \\
& \mathbb{C H} F_{n}^{*_{4}}=\left(F_{n}-i F_{n+1}\right)-\left(1-\frac{\left(F_{n+2}+i F_{n+3}\right) h}{F_{n}+i F_{n+1}}\right)
\end{aligned}
$$$$
\text { complex-hyperbolic conjugation, }
$$

$\mathbb{C H} F_{n}^{*_{5}}=\left(F_{n+2}+i F_{n+3}\right)-\left(F_{n}-i F_{n+1}\right) h$, anti-hyperbolic conjugation.

In this case, we can give the following relations:

$$
\begin{aligned}
& \mathbb{C H} F_{n}\left(\mathbb{C H} F_{n}\right)^{*_{1}}=F_{2 n+1}-F_{n+1} F_{n+4}+2 F_{n+2}\left(i F_{n+3}+h F_{n}+i h F_{n+1}\right), \\
& \mathbb{C H} F_{n}\left(\mathbb{C H} F_{n}\right)^{*_{2}}=-F_{n+2} F_{n-1}-F_{2 n+5}+2 F_{n+1}\left(i F_{n}+h F_{n+3}-i h F_{n+2}\right), \\
& \mathbb{C H} F_{n}\left(\mathbb{C H} F_{n}\right)^{*_{3}}=F_{2 n+1}+F_{n+1} F_{n+4}-2 F_{n+3}\left(i F_{n+2}+h F_{n+1}-i h F_{n}\right), \\
& \mathbb{C} \mathbb{H} F_{n}\left(\mathbb{C H} F_{n}\right)^{*_{4}}=F_{2 n+1}, \\
& \mathbb{C H} F_{n}\left(\mathbb{C H} F_{n}\right)^{*_{5}}=-h\left(F_{2 n+1}-F_{2 n+5}\right) .
\end{aligned}
$$

The norm of the circular-hyperbolic Fibonacci quaternions $\mathbb{C H} I F_{n}$ is defined in five different ways as follows:

$$
\begin{aligned}
N_{\mathbb{C H} F_{n}^{*_{1}}} & =\left\|\mathbb{C H} F_{n} \times\left(\mathbb{C H} F_{n}\right)^{*_{1}}\right\|^{2} \\
& =\left|F_{2 n+1}-F_{n+1} F_{n+4}+2 F_{n+2}\left(i F_{n+3}+h F_{n}+i h F_{n+1}\right)\right|, \\
N_{\mathbb{C H} F_{n}^{*_{2}}} & =\left\|\mathbb{C H} F_{n} \times\left(\mathbb{C H} F_{n}\right)^{*_{2}}\right\|^{2} \\
& =\left|-F_{n+2} F_{n-1}-F_{2 n+5}+2 F_{n+1}\left(i F_{n}+h F_{n+3}-i h F_{n+2}\right)\right|, \\
N_{\mathbb{C H} F_{n}^{* 3}} & =\left\|\mathbb{C H} F_{n} \times\left(\mathbb{C H} F_{n}\right)^{*_{3}}\right\|^{2} \\
& =\left|F_{2 n+1}+F_{n+1} F_{n+4}-2 F_{n+3}\left(i F_{n+2}+h F_{n+1}-i h F_{n}\right)\right|, \\
N_{\mathbb{C H} F_{n}^{*_{4}}} & =\left\|\mathbb{C H} F_{n} \times\left(\mathbb{C H} F_{n}\right)^{*_{4}}\right\|^{2} \\
& =F_{n}^{2}+F_{n+1}^{2}=F_{2 n+1}, \\
N_{\mathbb{C H} F_{n}^{* 5}} & =\left\|\mathbb{C H} F_{n} \times\left(\mathbb{C} \mathbb{H} F_{n}\right)^{*_{5}}\right\|^{2} \\
& =\left|-h\left(F_{2 n+1}-F_{2 n+5}\right)\right| .
\end{aligned}
$$


In the following theorem, some properties related to circular-hyperbolic Fibonacci quaternions are given.

Theorem 1. Let $F_{n}$ and $\mathbb{C H} F_{n}$ be the $n$-th terms of Fibonacci sequence $\left(F_{n}\right)$ and circularhyperbolic Fibonacci quaternion $\left(\mathbb{C H} F_{n}\right)$, respectively. In this case, for $n \geq 1$ we can give the following relations:

$$
\begin{gathered}
\mathbb{C H} F_{n}=\mathbb{C H} F_{n-1}+\mathbb{C H} F_{n-2}, \\
\mathbb{C H} F_{n+1}+\mathbb{C H} F_{n-1}=\mathbb{C H} L_{n}, \\
\mathbb{C H} F_{n+2}-\mathbb{C H} F_{n-2}=\mathbb{C H} L_{n}, \\
\mathbb{C H} F_{n}-i \mathbb{C H} F_{n+1}-h \mathbb{C H} F_{n+2}-i h \mathbb{C} \mathbb{H} F_{n+3}=F_{n}+F_{n+2}-F_{n+4}-F_{n+6} .
\end{gathered}
$$

Proof: Using (14) and (15), the proof can easily be done.

Theorem 2. (Honsberger identity) For $n, m \geq 0$ the Honsberger identity for the circularhyperbolic Fibonacci quaternions $\mathbb{C H} F_{n}$ and $\mathbb{C H} F_{m}$ is given by

$$
\begin{aligned}
\mathbb{C H} F_{n} \mathbb{C H} F_{m}+\mathbb{C H} F_{n+1} \mathbb{C H} F_{m+1}= & \mathbb{C H} F_{n+m+1}+2 F_{n+m+6} \\
& +i F_{n+m+2}+h F_{n+m+3}+i h F_{n+m+4} .
\end{aligned}
$$

Proof: By using (14) we get,

$$
\begin{aligned}
\mathbb{C H} F_{n} \mathbb{C H} F_{m}+\mathbb{C H} F_{n+1} \mathbb{C H} F_{m+1}= & \left(F_{n+m+1}-F_{n+m+3}+F_{n+m+5}+F_{n+m+7}\right) \\
& +2 i F_{n+m+2}+2 h F_{n+m+3}+2 i h F_{n+m+4} \\
= & \mathbb{C H} F_{n+m+1}+2 F_{n+m+6} \\
& +i F_{n+m+2}+h F_{n+m+3}+i h F_{n+m+4},
\end{aligned}
$$

where the identity $F_{n} F_{m}+F_{n+1} F_{m+1}=F_{n+m+1}$ is used $[10,14,24,25]$.

Theorem 3. (Generating function) Let $\mathbb{C H} F_{n}$ be the circular-hyperbolic Fibonacci quaternion. For the generating function for these quaternions is as follows:

$$
g_{\mathbb{C H} F_{n}}(t)=\sum_{s=0}^{n} \mathbb{C H} F_{n} t^{n}=\frac{\mathbb{C H} F_{0}+\left(\mathbb{C H} F_{1}-\mathbb{C H} F_{0}\right) t}{1-t-t^{2}} .
$$

Proof: Using the definition of generating function, we obtain

$$
g_{\mathbb{C H} F_{n}}(t)=\mathbb{C H} F_{0}+\mathbb{C H} F_{1} t+\ldots+\mathbb{C H} F_{n} t^{n}+\ldots
$$

Multiplying (1-t-t $\left.t^{2}\right)$ both sides of (41) and using (35), we have

$$
\left(1-t-t^{2}\right) g_{\mathbb{C H} F_{n}}(t)=\mathbb{C H} F_{0}+\left(\mathbb{C H} F_{1}-\mathbb{C H} F_{0}\right) t .
$$

Thus, the proof is completed.

Theorem 4. (Binet's Formula) Let $\mathbb{C} \mathbb{H} F_{n}$ be the circular-hyperbolic Fibonacci quaternion. For $n \geq 1$, Binet's formula for these quaternions is as follows:

$$
\mathbb{C} \mathbb{H} F_{n}=\frac{1}{\alpha-\beta}\left(\hat{\alpha} \alpha^{n}-\hat{\beta} \beta^{n}\right),
$$


where

$$
\hat{\alpha}=1+i \alpha+h \alpha^{2}+i h \alpha^{3}, \quad \alpha=\frac{1+\sqrt{5}}{2}
$$

and

$$
\hat{\beta}=1+i \beta+h \beta^{2}+i h \beta^{3}, \quad \beta=\frac{1-\sqrt{5}}{2} .
$$

Proof: Binet's formula of the circular-hyperbolic Fibonacci quaternion is the same as Binet's formula of the Fibonacci quaternion [8].

Theorem 5. (d'Ocagne's identity) For $n, m \geq 0$ the d'Ocagne's identity for the circularhyperbolic Fibonacci quaternions $\mathbb{C H} F_{n}$ and $\mathbb{C H} F_{m}$ is given by

$$
\begin{aligned}
\mathbb{C H} F_{m} \mathbb{C H} F_{n+1}-\mathbb{C H} F_{m+1} \mathbb{C H} F_{n}= & (-1)^{n} F_{m-n}(2+i+3 h+4 i h) \\
& +L_{m-n}(i-h+i h) .
\end{aligned}
$$

Proof: By using (14) we get,

$$
\begin{aligned}
\mathbb{C H} F_{m} \mathbb{C H} F_{n+1}-\mathbb{C H} F_{m+1} \mathbb{C H} F_{n}= & 2(-1)^{n} F_{m-n}+i(-1)^{n}\left(F_{m-n}+L_{m-n}\right) \\
& +h(-1)^{n}\left(3 F_{m-n}-L_{m-n}\right) \\
& +i h(-1)^{n}\left(4 F_{m-n}+L_{m-n}\right),
\end{aligned}
$$

where the identity $F_{n} F_{m}+F_{n+1} F_{m+1}=F_{n+m+1}, F_{n+3}-F_{n-3}=4 F_{n}$ and $F_{n+1}-F_{n-1}=L_{n}$ are used $[14,24,25]$.

Theorem 6. (Cassini's Identity) Let $\mathbb{C H} F_{n}$ be the circular-hyperbolic Fibonacci quaternion. For $n \geq 1$, Cassini's identity for $\mathbb{C H} F_{n}$ is as follows:

$$
\mathbb{C H} F_{n+1} \mathbb{C H} F_{n-1}-\mathbb{C H} F_{n}^{2}=(-1)^{n},(2+2 i+2 h+5 i h) .
$$

Proof: (44): By using (14) we get

$$
\begin{aligned}
\left.\mathbb{C H} F_{n+1} \mathbb{C H} F_{n-1}-\mathbb{C H} F_{n}\right)^{2}=[ & \left(F_{n+1} F_{n-1}-F_{n}^{2}\right)+\left(F_{n+1}^{2}-F_{n} F_{n+2}\right) \\
& \left.+\left(F_{n+3} F_{n+1}-F_{n+2}^{2}\right)+\left(F_{n+4} F_{n+2}-F_{n+3}^{2}\right)\right] \\
+ & i\left[\left(F_{n+2} F_{n-1}-F_{n+1} F_{n}\right)\right. \\
& \left.+\left(F_{n+4} F_{n+1}-F_{n+3} F_{n+2}\right)\right] \\
+ & h\left[\left(F_{n+1}^{2}-F_{n} F_{n+2}\right)\right. \\
& +\left(F_{n+1} F_{n+3}-F_{n+2}^{2}\right) \\
& +\left(F_{n+3} P_{n-1}-F_{n+2} F_{n}\right) \\
& \left.+\left(F_{n+4} F_{n}-F_{n+3} F_{n+1}\right)\right] \\
+ & i h\left[F_{n+1} F_{n+2}-F_{n} F_{n+1}\right) \\
& +\left(F_{n+4} F_{n-1}-F_{n+3} F_{n}\right) \\
& \left.+\left(F_{n+2} F_{n+1}-F_{n+3} F_{n}\right)\right] \\
= & -1)^{n}(2+2 i+2 h+5 i h) .
\end{aligned}
$$

where the identities of the Fibonacci numbers $F_{m} F_{n+1}-F_{m+1} F_{n}=(-1)^{n} F_{m-n}$ is used [14, $24,25]$. 
Theorem 7. (Catalan's Identity) Let $\mathbb{C H} F_{n}$ be the circular-hyperbolic Fibonacci quaternion. For $n \geq 1$, Catalan's identity for $\mathbb{C H} F_{n}$ is as follows:

$$
\begin{aligned}
& \mathbb{C H} F_{n}^{2}-\mathbb{C H} F_{n+r} \mathbb{C H} F_{n-r}=(-1)^{n-r} F_{r}\left[2 F_{r}+i\left(F_{r}+L_{r}\right)+h\left(3 F_{r}-L_{r}\right)\right. \\
& \left.+i h\left(4 F_{r}+L_{r}\right)\right] \text {. }
\end{aligned}
$$

Proof. By using (14), we get

$$
\begin{aligned}
\mathbb{C H} F_{n}^{2}-\mathbb{C H} F_{n+r} \mathbb{C H} F_{n-r}=[ & \left.F_{n}^{2}-F_{n+r} F_{n-r}\right)-\left(F_{n+1}^{2}-F_{n+r+1} F_{n-r+1}\right) \\
& +\left(F_{n+2}^{2}-F_{n+r+2} F_{n-r+2}\right) \\
& \left.+\left(F_{n+3}^{2}-F_{n+r+3} F_{n-r+3}\right)\right] \\
+ & i\left[\left(F_{n} F_{n+1}-F_{n+r} F_{n-r+1}\right)\right. \\
& +\left(F_{n+1} F_{n}-F_{n+r+1} F_{n-r}\right) \\
& -\left(F_{n+2} F_{n+3}-F_{n+r+2} F_{n-r+3}\right) \\
& \left.+\left(F_{n+3} F_{n+2}-F_{n+r+3} F_{n-r+2}\right)\right] \\
+ & h\left[\left(F_{n} F_{n+2}-F_{n+r} F_{n-r+2}\right)\right. \\
& -\left(F_{n+1} F_{n+3}-F_{n+r+1} F_{n-r+3}\right) \\
& +\left(F_{n+2} F_{n}-F_{n+r+2} F_{n-r}\right) \\
& \left.+\left(F_{n+3} F_{n+1}-F_{n+r+3} F_{n-r+1}\right)\right] \\
+ & i h\left[\left(F_{n} F_{n+3}-F_{n+r} F_{n-r+3}\right)\right. \\
& +\left(F_{n+1} F_{n+2}-F_{n+r+1} F_{n-r+2}\right) \\
& -\left(F_{n+2} F_{n+1}-F_{n+r+2} F_{n-r+1}\right) \\
& \left.+\left(F_{n+3} F_{n}-F_{n+r+3} F_{n-r}\right)\right] \\
= & -1)^{n-r} F_{r}\left[2 F_{r}+i\left(F_{r}+L_{r}\right)+h\left(3 F_{r}-L_{r}\right)\right. \\
& \left.+i h\left(4 F_{r}+L_{r}\right)\right] .
\end{aligned}
$$

where the identities of the Fibonacci numbers $F_{n-r} F_{n+r}-F_{n}^{2}=(-1)^{n-r+1} F_{r}^{2}$ and $F_{m} F_{n}-F_{m+r} F_{n-r}=(-1)^{n-r} F_{m+r-n} F_{r}$ are used [24].

\section{Conclusion}

In this study, a number of new results on circular-hyperbolic Fibonacci quaternions were derived.

\section{References}

[1] Bodnar, O. Y. (1994). The Golden Section and Non-Euclidean Geometry in Nature and Art, Publishing House "Svit", Lvov (In Russian).

[2] Catoni, F., Boccaletti, R., Cannata, R., Catoni, V., Nichelatti, E., \& Zampatti, P. (2008). The Mathematics of Minkowski Space-Time, Birkhauser, Basel.

[3] Cihan, A., Azak, A. Z., Güngör, M. A., \& Tosun, M. (2019). A study of Dual Hyperbolic Fibonacci and Lucas numbers. An. St. Univ. Ovidius Constanta, 27 (1), 35-48. 
[4] Clifford, W. K. (1873). Preliminary sketch of bi-quaternions. Proc. London Math. Soc., 64 (4), 381-395.

[5] Dattoli, G., Licciardi, S., Pidatella, R. M., \& Sabia, E. (2018). Hybrid complex numbers: The matrix version, Adv. Appl. Clifford Algebras, 28 (3), 58.

[6] Gargoubi, H. \& Kossentini, S. (2016). f-algebra structure on hyperbolic numbers. Adv. Appl. Clifford Algebras, 26 (4), 1211-1233.

[7] Güngör, M. A., \& Azak, A. Z. (2017). Investigation of dual complex Fibonacci, dual complex Lucas numbers and their properties, Adv. Appl. Clifford Algebras, 27 (4), 3083-3096.

[8] Halıcı, S. (2012). On Fibonacci Quaternions, Adv. Appl. Clifford Algebras, 22 (2), 321-327.

[9] Hamilton, W. R. (1866). Elements of Quaternions. Longmans, Green and Co., London.

[10] Horadam, A. F. (1963). Complex Fibonacci Numbers and Fibonacci Quaternions, American Math. Monthly, 70 (3), 289-291.

[11] Horadam, A. F. (1993). Quaternion Recurrence Relations, Ulam Quarterly, 2 (2), 23-33.

[12] Iyer, M. R. (1969). A Note on Fibonacci Quaternions, The Fibonacci Quarterly, 7 (3), 225-229.

[13] Iyer, M. R. (1969). Some Results on Fibonacci Quaternions, The Fibonacci Quaterly, 7, 201-210.

[14] Koshy, T. (2001) Fibonacci and Lucas Numbers with Applications, John Wiley and Sons, Proc., New York-Toronto.

[15] Majernik, V. (2006) Quaternion formulation of the Galilean space-time transformation, Acta Phy. Slovaca, 56 (1), 9-14.

[16] Majernik, V. (1996). Multicomponent number systems, Acta Physica. Polonica A, 90 (3), 491-498.

[17] Messelmi, F. (2015). Dual complex numbers and their holomorphic functions, working paper or preprint (Jan. 2015).

[18] Motter, A. E., \& Rosa, A. F. (1998). Hyperbolic calculus, Adv. Appl. Clifford Algebras, 8 (1), 109-128.

[19] Nurkan, K. S., \& Güven, A. İ. (2015). Dual Fibonacci Quaternions, Adv. Appl. Clifford Algebras, 25 (2), 403-414.

[20] Nurkan, K. S., \& Güven, A. İ. (2015). A New Approach to Fibonacci, Lucas numbers and dual vectors, Adv. Appl. Clifford Algebras, 25 (3), 577-590. 
[21] Pennestri, E., \& Stefanelli, R. (2007). Linear algebra and numerical algorithms using dual numbers. Multibody Syst, 18 (3), 323-344.

[22] Stakhov, A. P., \& Tkachenko, I. S. (2005). The golden shofar, Chaos Solitons Fractals, $26(3), 677-684$.

[23] Stakhov, A. P., \& Tkachenko, I. S. (1993). Hyperbolic Fibonacci trigonometry, Reports of the National Academy of Sciences of Ukraine, 208 (7), 9-14.

[24] Vajda, S. (1989). Fibonacci and Lucas Numbers, and the Golden Section, Ellis Horwood Limited Publ., England.

[25] Verner, E., \& Hoggatt, Jr. (1969). Fibonacci and Lucas Numbers. The Fibonacci Association.

[26] Ollerton, R. L., \& Shannon, A. G. (1992). An extension of circular and hyperbolic functions, International Journal of Mathematical Education in Science and Technology, $23(4), 611-620$. 